1 Hacettepe Journal of Mathematics and Statistics

$\bigcap$ Volume 46 (2) (2017), 165-176

\title{
New formulae of squares of some Jacobi polynomials via hypergeometric functions
}

\author{
W.M. Abd- Elhameed* ${ }^{\dagger}$
}

\begin{abstract}
In this article, a new formula expressing explicitly the squares of Jacobi polynomials of certain parameters in terms of Jacobi polynomials of arbitrary parameters is derived. The derived formula is given in terms of ceratin terminating hypergeometric function of the type ${ }_{4} F_{3}(1)$. In some cases, this ${ }_{4} F_{3}(1)$ can be reduced by using some well-known reduction formulae in literature such as Watson's and Pfaff-Saalschütz's identities. In some other cases, this ${ }_{4} F_{3}(1)$ can be reduced by means of symbolic computation, and in particular Zeilberger's, Petkovsek's and van Hoeij's algorithms. Hence, some new squares formulae for Jacobi polynomials of special parameters can be deduced in reduced forms which are free of any hypergeometric functions.
\end{abstract}

Keywords: Jacobi polynomials; linearization coefficients; generalized hypergeometric functions; computer algebra, standard reduction formulae

2000 AMS Classification: 33F10; 33C20; 33Cxx; 68W30

Received : 23.02.2016 Accepted : 19.05.2016 Doi : 10.15672/HJMS.20164518618

\section{Introduction}

The Jacobi polynomials are of fundamental importance in theoretical and applied mathematical analysis. The class of Jacobi polynomials contains six well-known families of orthogonal polynomials, they are, ultraspherical, Legendre and the four kinds of Chebyshev polynomials. The Jacobi polynomials in general and their six special polynomials in particular are extensively employed in obtaining numerical solutions of ordinary, fractional and partial differential equations. In this respect, these polynomials are employed for the sake of obtaining spectral solutions for various kinds of differential equations. For example, Abd-Elhameed in [1] has employed Legendre polynomials for solving linear and nonlinear sixth-order two point boundary value problems via an elegant harmonic numbers operational matrix of derivatives. Also, Chebyshev, ultraspherical and

*Department of Mathematics, Faculty of Science, Cairo University, Giza, Egypt, Email: walee_9@yahoo.com

$\dagger$ Corresponding Author. 
Jacobi polynomials are used for solving various kinds of boundary value problems (see, for example [16, 5, 12]).

The two problems of linearizing products of various orthogonal polynomials and the connection coefficients between them are of interest. There are several old and recent studies in this direction. For example, the two problems concerning linearization and connection coefficients of ultraspherical and Jacobi polynomials have been studied in several articles, see for instance $[7,18,19,20,26,9]$. Some other investigations for the problems of the linearization and connection coefficients for various orthogonal polynomials can be found in $[10,11,3,2,4,15,23,28,29]$. Recently, the problems of connection, duplication and linearization coefficients of various orthogonal polynomials have been investigated in the Ph.D. thesis of Tcheutia [30].

To be more precise, and if we consider the two polynomials $F_{m}(x)$ and $G_{n}(x)$ of degrees $m$ and $n$ respectively, then the linearization problem requires to find the linearization coefficients $L_{m, n, k}$ such that

$$
F_{m}(x) G_{n}(x)=\sum_{k=0}^{m+n} L_{m, n, k} Q_{k}(x)
$$

and $\left\{Q_{k}(x)\right\}_{k \geq 0}$ is an arbitrary sequence of orthogonal polynomials.

The following two special problems of (1.1) are of interest:

: (i) Clebsch-Gordan-type problem which requires to find the coefficients $\xi_{m, n, k}$ such that

$$
F_{m}(x) F_{n}(x)=\sum_{k=0}^{m+n} \xi_{m, n, k} F_{k}(x) .
$$

: (ii) The connection problem, which requires to find the coefficients $\gamma_{m, k}$, such that

$$
F_{m}(x)=\sum_{k=0}^{m} \gamma_{m, k} Q_{k}(x)
$$

The principal aim of this article is to establish some new formulae for the squares of Jacobi polynomials of certain parameters. In particular, we find the coefficients $L_{m, n, k}$ in (1.1) for the case corresponds to: $m=n, F_{m}(x)=G_{n}(x)=P_{m}^{\left(\alpha,-\frac{1}{2}\right)}(x)$, and $Q_{k}(x)=$ $P_{k}^{(\gamma, \delta)}(x)$.

The rest of the paper is as follows. In Section 2, some mathematical preliminaries concerning some properties of the classical Jacobi polynomials are presented. Moreover, some transformation formulae between certain hypergeometric functions and also some standard formulae of ceratin hypergeometric functions are presented. In Section 3, we derive in detail a theorem in which a new squares formula of certain Jacobi polynomials is given in terms of Jacobi polynomials of arbitrary parameters. Moreover, in this section, and based on applying some standard formulae, some new linearization formulae are given in explicit forms free of any hypergeometric functions. In Section 4, we describe how symbolic computation, and in particular, the algorithms of Zeilberger, Petkovsek and van Hoeij can be utilized for obtaining some reduced linearization formulae of Jacobi polynomials of particular parameters. 


\section{Preliminaries and useful transformations}

This section is dedicated to presenting some properties of the classical Jacobi polynomials. Moreover, some important transformation formulae between some types of hypergeometric functions and also some standard reduction formulae of certain hypergeometric functions are displayed.

2.1. Some relevant properties of the classical Jacobi polynomials. The classical Jacobi polynomials associated with the real parameters $(\gamma>-1, \delta>-1)$ (see, Olver et al. [25], Andrews et al. [6] and Rainville [27]), are a sequence of polynomials $P_{m}^{(\gamma, \delta)}(x), x \in$ $[-1,1](m=0,1,2, \ldots)$, each respectively of degree $m$. From now on, we use the following normalized Jacobi polynomials introduced by Rahman [26] and used in [14].

$$
\begin{aligned}
R_{m}^{(\gamma, \delta)}(x) & =\frac{P_{m}^{(\gamma, \delta)}(x)}{P_{m}^{(\gamma, \delta)}(1)}=\frac{m !}{(\gamma+1)_{m}} P_{m}^{(\gamma, \delta)}(x) \\
& ={ }_{2} F_{1}\left(\begin{array}{c|c}
-m, m+\gamma+\delta+1 & \frac{1-x}{2}
\end{array}\right) .
\end{aligned}
$$

The polynomials $R_{m}^{(\gamma, \delta)}(x)$ satisfy the following orthogonality relation:

$$
\int_{-1}^{1}(1-x)^{\gamma}(1+x)^{\delta} R_{m}^{(\gamma, \delta)}(x) R_{n}^{(\gamma, \delta)}(x) d x= \begin{cases}0, & m \neq n \\ h_{m}^{(\gamma, \delta)}, & m=n\end{cases}
$$

where

$$
h_{m}^{(\gamma, \delta)}=\frac{2^{\gamma+\delta+1} m ! \Gamma(m+\delta+1)[\Gamma(\gamma+1)]^{2}}{(2 m+\gamma+\delta+1) \Gamma(m+\gamma+\delta+1) \Gamma(m+\gamma+1)} .
$$

The advantage of using the normalized Jacobi polynomials in (2.1) is that its use yields directly the following six polynomials as special cases.

$$
\begin{array}{rlrl}
C_{m}^{(\alpha)}(x) & =R_{m}^{\left(\alpha-\frac{1}{2}, \alpha-\frac{1}{2}\right)}(x), & & T_{m}(x)=R_{m}^{\left(-\frac{1}{2},-\frac{1}{2}\right)}(x), \\
U_{m}(x) & =(m+1) R_{m}^{\left(\frac{1}{2}, \frac{1}{2}\right)}(x), & & V_{m}(x)=R_{m}^{\left(-\frac{1}{2}, \frac{1}{2}\right)}(x), \\
W_{m}(x)=(2 m+1) R_{m}^{\left(\frac{1}{2},-\frac{1}{2}\right)}(x), & P_{m}(x)=R_{m}^{(0,0)}(x),
\end{array}
$$

where $C_{i}^{(\alpha)}(x), T_{i}(x), U_{i}(x), V_{i}(x), W_{i}(x)$ and $P_{i}(x)$ are the ultraspherical, Chebyshev of the first, second, third and fourth kinds, and Legendre polynomials, respectively.

The following identity is also important.

$$
R_{m}^{(\gamma, \delta)}(-x)=\frac{(-1)^{m} \Gamma(\gamma+1) \Gamma(m+\delta+1)}{\Gamma(\delta+1) \Gamma(m+\gamma+1)} R_{m}^{(\delta, \gamma)}(x) .
$$

For more properties on Jacobi polynomials in general and their special polynomials in particular, one can be referred to the important books of Andrews et al. [6] and Mason and Handscomb. [24].

2.2. Some Transformation formulae. Recall the following well-known definition of the generalized hypergeometric function

$$
{ }_{p} F_{q}\left(\begin{array}{c}
a_{1}, a_{2} \ldots, a_{p} \\
b_{1}, b_{2} \ldots, b_{q}
\end{array} \mid x\right)=\sum_{j=0}^{\infty} \frac{\left(a_{1}\right)_{j}\left(a_{2}\right)_{j} \ldots\left(a_{p}\right)_{j}}{\left(b_{1}\right)_{j}\left(b_{2}\right)_{j} \ldots\left(b_{q}\right)_{j}} \frac{x^{j}}{j !},
$$

where $a_{1}, a_{2}, \ldots, a_{p}, b_{1}, b_{2}, \ldots, b_{q}$, are complex or real parameters, with $b_{i} \neq 0$, for all $1 \leq i \leq q$.

The following five theorems are of fundamental importance in establishing our results. 
2.1. Theorem. Let $m, p, q, r, s, t, u$ be nonnegative integers. The following transformation formula holds (see, [17] and [22])

$$
\begin{aligned}
& { }_{p+r+1} F_{q+s}\left(\begin{array}{c}
-m,\left[a_{p}\right],\left[c_{r}\right] \\
{\left[b_{q}\right],\left[d_{s}\right]}
\end{array} \mid y z\right)=\sum_{j=0}^{m}\left(\begin{array}{c}
m \\
j
\end{array}\right) \frac{\left(a_{p}\right)_{j}\left(\alpha_{t}\right)_{j} y^{j}}{\left(b_{q}\right)_{j}\left(\beta_{u}\right)_{j}(j+\mu)_{j}} \times \\
& { }_{p+t+1} F_{q+u+1}\left(\begin{array}{c}
j-m,\left[j+a_{p}\right],\left[j+\alpha_{t}\right] \\
2 j+\mu+1,\left[j+b_{q}\right],\left[j+\beta_{u}\right]
\end{array} \mid y\right) \times \\
& { }_{r+u+2} F_{s+t}\left(\begin{array}{c}
-j, j+\mu,\left[c_{r}\right],\left[\beta_{u}\right] \\
{\left[d_{s}\right],\left[\alpha_{t}\right]}
\end{array}\right) .
\end{aligned}
$$

2.2. Theorem. The following transformation formula holds (see, [8])

$$
\begin{aligned}
& { }_{2} F_{1}\left(\begin{array}{c}
a, b \\
a+b-\frac{1}{2}
\end{array} \mid y\right){ }_{2} F_{1}\left(\begin{array}{c}
a, b \\
a+b+\frac{1}{2}
\end{array} \mid y\right)= \\
& { }_{3} F_{2}\left(\begin{array}{c}
2 a, 2 b, a+b \\
2 a+2 b-1, a+b+\frac{1}{2}
\end{array} \mid y\right) .
\end{aligned}
$$

2.3. Theorem. Pfaff-Saalschütz identity (see, [25])

For every nonnegative integer $m$, and for $a_{2}+b_{2}=a_{1}+b_{1}+1-m$, one has

$$
{ }_{3} F_{2}\left(\begin{array}{c|c}
-m, a_{1}, b_{1} \\
a_{2}, b_{2}
\end{array} \mid 1\right)=\frac{\left(a_{2}-a_{1}\right)_{m}\left(a_{2}-b_{1}\right)_{m}}{\left(a_{2}\right)_{m}\left(a_{2}-a_{1}-b_{1}\right)_{m}} .
$$

2.4. Theorem. Watson's identity (see, [32])

$$
\begin{aligned}
& { }_{3} F_{2}\left(\begin{array}{c|c}
-m, m+2 a+2 b-1, a \\
2 a, a+b
\end{array}\right) \\
& = \begin{cases}\frac{m ! \Gamma\left(a+\frac{m}{2}\right) \Gamma\left(b+\frac{m}{2}\right) \Gamma(2 a) \Gamma(a+b)}{\left(\frac{m}{2}\right) ! \Gamma\left(a+b+\frac{m}{2}\right) \Gamma(2 a+m) \Gamma(a) \Gamma(b)}, & m \text { even }, \\
0, & \text { m odd. }\end{cases}
\end{aligned}
$$

2.5. Theorem. The following identity (Clausen's identity) holds (see, [27])

$$
{ }_{3} F_{2}\left(\begin{array}{c}
2 a, 2 b, a+b \\
2 a+2 b, a+b+\frac{1}{2}
\end{array} \mid y\right)=\left[{ }_{2} F_{1}\left(\begin{array}{c|c}
a, b \\
a+b+\frac{1}{2}
\end{array} \mid y\right)\right]^{2} .
$$

\section{Squares formulae of certain Jacobi polynomials}

This section is devoted to establishing new linearization formulae of products of particular Jacobi polynomials. We will state and prove a new theorem, in which a new formula of squares of certain Jacobi polynomials is expressed in terms of Jacobi polynomials of arbitrary parameters.

3.1. Theorem. For every nonnegative integer $n$, the following linearization formula holds:

$$
\begin{aligned}
& \left(R_{n}^{\left(\alpha,-\frac{1}{2}\right)}(x)\right)^{2}=\sum_{j=0}^{2 n} \frac{\left(\begin{array}{c}
2 n \\
j
\end{array}\right)\left(\alpha+\frac{1}{2}\right)_{j}(\gamma+1)_{j}(2 n+2 \alpha+1)_{j}}{(\alpha+1)_{j}(2 \alpha+1)_{j}(j+\gamma+\delta+1)_{j}} \times \\
& { }_{4} F_{3}\left(\begin{array}{c}
j-2 n, j+\alpha+\frac{1}{2}, j+2 \alpha+2 n+1, j+\gamma+1 \\
j+\alpha+1, j+2 \alpha+1,2 j+\gamma+\delta+2
\end{array} \mid 1\right) R_{j}^{(\gamma, \delta)}(x) .
\end{aligned}
$$


Proof. Making use of the Gauss hypergeometric representation of the orthogonal polynomials $R_{n}^{\left(\alpha,-\frac{1}{2}\right)}(x)$, one can write

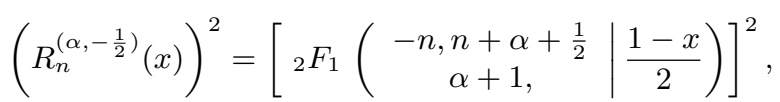

and in virtue of the Clausen's identity (2.9), relation (3.2) can be turned into

$$
\left(R_{n}^{\left(\alpha,-\frac{1}{2}\right)}(x)\right)^{2}={ }_{3} F_{2}\left(\begin{array}{c|c}
-2 n, 2 n+2 \alpha+1, \alpha+\frac{1}{2} & \frac{1-x}{2} \\
2 \alpha+1, \alpha+1
\end{array}\right) .
$$

If Theorem 2.1 is applied to the right hand side of (3.3), with the following choices:

$$
p=q=2, r=s=u=0, t=1,\left[c_{r}\right]=\left[d_{s}\right]=\left[\beta_{u}\right]=\emptyset, y=1, z=\frac{1-x}{2},
$$

and if we choose the remaining parameters suitably, then the following transformation formula holds:

$$
\begin{aligned}
& { }_{3} F_{2}\left(\begin{array}{c|c}
-2 n, 2 n+2 \alpha+1, \alpha+\frac{1}{2} & 1-x \\
2 \alpha+1, \alpha+1
\end{array}\right)= \\
& \sum_{j=0}^{2 n} \frac{\left(\begin{array}{c}
2 n \\
j
\end{array}\right)\left(\alpha+\frac{1}{2}\right)_{j}(\gamma+1)_{j}(2 n+2 \alpha+1)_{j}}{(\alpha+1)_{j}(2 \alpha+1)_{j}(j+\gamma+\delta+1)_{j}} \times \\
& { }_{4} F_{3}\left(\begin{array}{c}
j-2 n, j+\alpha+\frac{1}{2}, j+2 \alpha+2 n+1, j+\gamma+1 \\
j+\alpha+1, j+2 \alpha+1,2 j+\gamma+\delta+2
\end{array}\right. \\
& { }_{2} F_{1}\left(\begin{array}{c}
-j, j+\gamma+\delta+1 \\
\gamma+1,
\end{array} \mid \frac{1-x}{2}\right),
\end{aligned}
$$

and this in turn leads to the following linearization formula:

$$
\begin{aligned}
& \left(R_{n}^{\left(\alpha,-\frac{1}{2}\right)}(x)\right)^{2}=\sum_{j=0}^{2 n} \frac{\left(\begin{array}{c}
2 n \\
j
\end{array}\right)\left(\alpha+\frac{1}{2}\right)_{j}(\gamma+1)_{j}(2 n+2 \alpha+1)_{j}}{(\alpha+1)_{j}(2 \alpha+1)_{j}(j+\gamma+\delta+1)_{j}} \times \\
& { }_{4} F_{3}\left(\begin{array}{c}
j-2 n, j+\alpha+\frac{1}{2}, j+2 \alpha+2 n+1, j+\gamma+1 \\
j+\alpha+1, j+2 \alpha+1,2 j+\gamma+\delta+2
\end{array} \mid 1\right) R_{j}^{(\gamma, \delta)}(x) .
\end{aligned}
$$

Theorem 3.1 is proved.

3.2. Corollary. If we set $\gamma=\alpha$ and $\delta=\frac{-1}{2}$ in formula (3.1), then the following linearization formula is obtained:

$$
\begin{aligned}
\left(R_{n}^{\left(\alpha,-\frac{1}{2}\right)}(x)\right)^{2}= & \sum_{j=0}^{2 n} \frac{\left(\begin{array}{c}
2 n \\
j
\end{array}\right)(j+1)_{2 n-j}\left(\alpha+\frac{1}{2}\right)_{j}\left(j-2 n-\alpha+\frac{1}{2}\right)_{2 n-j}}{\left(j+\alpha+\frac{1}{2}\right)_{j}(2 \alpha+1)_{j}\left(2 j+\alpha+\frac{3}{2}\right)_{2 n-j}} \times \\
& \frac{(2 n+2 \alpha+1)_{j}}{(-2(n+\alpha))_{2 n-j}} R_{j}^{\left(\alpha,-\frac{1}{2}\right)}(x) .
\end{aligned}
$$

Proof. The substitution by $\gamma=\alpha$ and $\delta=-\frac{1}{2}$ into relation (3.1) yields

$$
\begin{aligned}
& \left(R_{n}^{\left(\alpha,-\frac{1}{2}\right)}(x)\right)^{2}=\sum_{j=0}^{2 n} \frac{\left(\begin{array}{c}
2 n \\
j
\end{array}\right)\left(\alpha+\frac{1}{2}\right)_{j}(2 n+2 \alpha+1)_{j}}{\left(j+\alpha+\frac{1}{2}\right)_{j}(2 \alpha+1)_{j}} \times \\
& { }_{3} F_{2}\left(\begin{array}{c|c}
j-2 n, j+\alpha+\frac{1}{2}, j+2 \alpha+2 n+1 & 1 \\
2 j+\alpha+\frac{3}{2}, j+2 \alpha+1 & 1
\end{array}\right) R_{j}^{\left(\alpha,-\frac{1}{2}\right)}(x) .
\end{aligned}
$$


The ${ }_{3} F_{2}(1)$ in (3.5) is balanced, and it can be summed by means of Pfaff-Saalschütz identity (2.7) to give

$$
\begin{aligned}
& { }_{3} F_{2}\left(\begin{array}{c|c}
j-2 n, j+\alpha+\frac{1}{2}, j+2 \alpha+2 n+1 & 1 \\
2 j+\alpha+\frac{3}{2}, j+2 \alpha+1 & 1
\end{array}\right)= \\
& \frac{(j+1)_{2 n-j}\left(j-2 n-\alpha+\frac{1}{2}\right)_{2 n-j}}{\left(2 j+\alpha+\frac{3}{2}\right)_{2 n-j}(-2(n+\alpha))_{2 n-j}} .
\end{aligned}
$$

The last reduction formula turns formula (3.5) to the following simplified linearization formula

$$
\begin{aligned}
\left(R_{n}^{\left(\alpha,-\frac{1}{2}\right)}(x)\right)^{2}= & \sum_{j=0}^{2 n} \frac{\left(\begin{array}{c}
2 n \\
j
\end{array}\right)(j+1)_{2 n-j}\left(\alpha+\frac{1}{2}\right)_{j}\left(j-2 n-\alpha+\frac{1}{2}\right)_{2 n-j}}{\left(j+\alpha+\frac{1}{2}\right)_{j}(2 \alpha+1)_{j}\left(2 j+\alpha+\frac{3}{2}\right)_{2 n-j}} \times \\
& \frac{(2 n+2 \alpha+1)_{j}}{(-2(n+\alpha))_{2 n-j}} R_{j}^{\left(\alpha,-\frac{1}{2}\right)}(x) .
\end{aligned}
$$

Corollary 3.2 is proved.

3.3. Corollary. The two formulae expressing explicitly the squares of Chebyshev polynomials of fourth and third kinds in terms of their original polynomials can be obtained as special cases of (3.4).

$$
\begin{aligned}
& W_{n}^{2}(x)=\sum_{j=0}^{2 n} W_{j}(x), \\
& V_{n}^{2}(x)=\sum_{j=0}^{2 n}(-1)^{j} V_{j}(x) .
\end{aligned}
$$

3.4. Corollary. The two formulae (3.6) and (3.7) are in complete agreement with those obtained in [13], but they are derived here differently.

3.5. Corollary. If we set $\alpha=-\frac{1}{2}$ and $\delta=\gamma=\beta-\frac{1}{2}$, in formulae (3.1), then the following linearization formula is obtained:

$$
\begin{aligned}
T_{n}^{2}(x) & =\frac{1}{2}+\frac{(-1)^{n}(\Gamma(\beta+1))^{2}}{2 \Gamma(-n+\beta+1) \Gamma(n+\beta+1)}+\frac{\pi \Gamma(\beta+1)}{(2 n-1) ! \Gamma\left(\beta+\frac{1}{2}\right)} \times \\
& \sum_{j=1}^{2 n} \frac{2^{-2 \beta-j+2 n-1}\left(\begin{array}{c}
2 n \\
j
\end{array}\right)(\beta+j) \Gamma(j+2 \beta) \Gamma\left(\frac{j}{2}+n\right)}{\Gamma\left(\frac{1}{2}(j-2 n+1)\right) \Gamma\left(\frac{j}{2}-n+\beta+1\right) \Gamma\left(\frac{j}{2}+n+\beta+1\right)} C_{j}^{(\beta)}(x) .
\end{aligned}
$$

Proof. First, with the aid of the Legendre's duplication formula

$$
\frac{\Gamma(2 z)}{\Gamma(z)}=\frac{2^{2 z-1} \Gamma\left(z+\frac{1}{2}\right)}{\sqrt{\pi}},
$$

it is not difficult to see that

$$
\lim _{\alpha \rightarrow \frac{-1}{2}} \frac{\left(\alpha+\frac{1}{2}\right)_{k}}{(2 \alpha+1)_{k}}= \begin{cases}1 & k=0, \\ \frac{1}{2} & k \geq 1 .\end{cases}
$$

If we set $\alpha=-\frac{1}{2}$ and $\delta=\gamma=\beta-\frac{1}{2}$ in (3.1), and if we take into account the limit in (3.9), then we get 


$$
\begin{aligned}
T_{n}^{2}(x)= & \frac{1}{2}+\frac{1}{2} \sum_{j=0}^{2 n} \frac{\left(\begin{array}{c}
2 n \\
j
\end{array}\right)(2 n)_{j}\left(\beta+\frac{1}{2}\right)_{j}}{\left(\frac{1}{2}\right)_{j}(j+2 \beta)_{j}} \times \\
& { }_{3} F_{2}\left(\begin{array}{c}
j-2 n, j+2 n, \beta+j+\frac{1}{2} \\
j+\frac{1}{2}, 2 \beta+2 j+1
\end{array} \mid 1\right) C_{j}^{(\beta)}(x) .
\end{aligned}
$$

Making use of Watson's identity (2.8), the ${ }_{3} F_{2}(1)$ in (3.10) reduces to

$$
\begin{aligned}
& { }_{3} F_{2}\left(\begin{array}{c}
j-2 n, j+2 n, \beta+j+\frac{1}{2} \\
j+\frac{1}{2}, 2 \beta+2 j+1
\end{array} \mid \begin{array}{cc}
\sqrt{\pi} \Gamma(\beta+1) \Gamma\left(j+\frac{1}{2}\right) \Gamma(j+\beta+1) \\
\frac{\sqrt{2}+2 n+1}{\Gamma\left(\frac{j-1}{2}\right) \Gamma\left(\frac{j+1}{2}+n\right) \Gamma\left(\frac{j}{2}-n+\beta+1\right) \Gamma\left(\frac{j}{2}+n+\beta+1\right)}, & j \text { even, } \\
0, & j \text { odd. }
\end{array}\right.
\end{aligned}
$$

With the aid of the last reduction formula, and after performing some manipulations, linearization formula (3.10) is turned into

$$
\begin{aligned}
T_{n}^{2}(x) & =\frac{1}{2}+\frac{(-1)^{n}(\Gamma(\beta+1))^{2}}{2 \Gamma(-n+\beta+1) \Gamma(n+\beta+1)}+\frac{\pi \Gamma(\beta+1)}{(2 n-1) ! \Gamma\left(\beta+\frac{1}{2}\right)} \times \\
& \sum_{j=1}^{2 n} \frac{2^{-2 \beta-j+2 n-1}\left(\begin{array}{c}
2 n \\
j
\end{array}\right)(\beta+j) \Gamma(j+2 \beta) \Gamma\left(\frac{j}{2}+n\right)}{\Gamma\left(\frac{1}{2}(j-2 n+1)\right) \Gamma\left(\frac{j}{2}-n+\beta+1\right) \Gamma\left(\frac{j}{2}+n+\beta+1\right)} C_{j}^{(\beta)}(x) .
\end{aligned}
$$

Corollary 3.5 is proved.

3.6. Remark. It is worthy to note here that formula (3.8) leads to the same relation obtained by (Sanchez [28], formula 21, page 265), taking into account the identity

$$
C_{n}^{(\alpha)}(x)=\frac{n !}{(2 \alpha)_{n}} \bar{C}_{n}^{(\alpha)}(x),
$$

where $\bar{C}_{n}^{(\alpha)}(x)$ is the Gegenbauer polynomial of degree $n$ which used in Ref. [28].

3.7. Corollary. If we set $\alpha=\delta=-\frac{1}{2}$ in formulae (3.1), then the following linearization formula is obtained:

$$
\begin{aligned}
T_{n}^{2}(x)=\frac{1}{2}+\frac{1}{2} & \sum_{j=0}^{2 n} \frac{(-1)^{j}\left(\begin{array}{c}
2 n \\
j
\end{array}\right)(2 n)_{j}(\gamma+1)_{j}\left(j-2 n+\gamma+\frac{3}{2}\right)_{2 n-j}}{\left(\frac{1}{2}\right)_{j}\left(j+\gamma+\frac{1}{2}\right)_{j}\left(2 j+\gamma+\frac{3}{2}\right)_{2 n-j}} \times \\
& R_{j}^{\left(\gamma,-\frac{1}{2}\right)}(x)
\end{aligned}
$$

Proof. The substitution by $\alpha=\delta=-\frac{1}{2}$ into relation (3.1) yields

$$
\begin{aligned}
& T_{n}^{2}(x)=\frac{1}{2}+\frac{1}{2} \sum_{j=0}^{2 n} \frac{\left(\begin{array}{c}
2 n \\
j
\end{array}\right)(2 n)_{j}(\gamma+1)_{j}}{\left(\frac{1}{2}\right)_{j}\left(j+\gamma+\frac{1}{2}\right)_{j}} \times \\
&{ }_{3} F_{2}\left(\begin{array}{c}
j-2 n, j+2 n, j+\gamma+1 \\
j+\frac{1}{2}, 2 j+\gamma+\frac{3}{2}
\end{array}\right.1) R_{j}^{\left(\gamma,-\frac{1}{2}\right)}(x) .
\end{aligned}
$$

The ${ }_{3} F_{2}(1)$ in $(3.12)$ can be reduced by means of Pfaff-Saalschütz identity (2.7) to the form

$$
{ }_{3} F_{2}\left(\begin{array}{c|c}
j-2 n, j+2 n, j+\gamma+1 \\
j+\frac{1}{2}, 2 j+\gamma+\frac{3}{2}
\end{array} \mid 1\right)=\frac{(-1)^{j}\left(j-2 n+\gamma+\frac{3}{2}\right)_{2 n-j}}{\left(2 j+\gamma+\frac{3}{2}\right)_{2 n-j}}
$$


and this in turn yields the following linearization formula

$$
\begin{gathered}
T_{n}^{2}(x)=\frac{1}{2}+\frac{1}{2} \sum_{j=0}^{2 n} \frac{(-1)^{j}\left(\begin{array}{c}
2 n \\
j
\end{array}\right)(2 n)_{j}(\gamma+1)_{j}\left(j-2 n+\gamma+\frac{3}{2}\right)_{2 n-j}}{\left(\frac{1}{2}\right)_{j}\left(j+\gamma+\frac{1}{2}\right)_{j}\left(2 j+\gamma+\frac{3}{2}\right)_{2 n-j}} \times \\
R_{j}^{\left(\gamma,-\frac{1}{2}\right)}(x) .
\end{gathered}
$$

\section{Use of Computer algebra algorithms in developing some other new linearization formulae}

In this section, we are interested in developing some other new squares formulae of certain parameters Jacobi polynomials. To be more precise, the $4 F_{3}(1)$ in relation (3.1) can be reduced in some cases by making use of some standard reduction formulae such as Pfaff-Saalschütz's and Watson's identities, however in other cases this reduction is not available. So, we resorted to some computer algebra algorithms to reduce this $4 F_{3}(1)$ of certain choices of the involved parameters in formula (3.1).

4.1. Corollary. If we set $\alpha=-\frac{1}{2}, \delta=\frac{1}{2}$ in formula (3.1), then the following linearization formula is obtained:

$$
\begin{aligned}
T_{n}^{2}(x)=\frac{1}{2}+\frac{1}{4} \sum_{j=0}^{2 n} \frac{(-1)^{j}\left(\begin{array}{c}
2 n \\
j
\end{array}\right) \Gamma\left(\gamma+\frac{3}{2}\right)(2 n)_{j}(\gamma+1)_{j}}{(2 j+1)\left(\frac{1}{2}\right)_{j}\left(j+\gamma+\frac{3}{2}\right)_{j}\left(2 j+\gamma+\frac{5}{2}\right)_{2 n-j}} \times \\
\frac{\left(2 \gamma+2 j(2 \gamma+2 j+3)-16 n^{2}+3\right)}{\Gamma\left(j-2 n+\gamma+\frac{5}{2}\right)} R_{j}^{\left(\gamma, \frac{1}{2}\right)}(x) .
\end{aligned}
$$

Proof. Substituting $\alpha=-\frac{1}{2}, \delta=\frac{1}{2}$ into relation (3.1) leads to the linearization formula

$$
\begin{aligned}
T_{n}^{2}(x)=\frac{1}{2}+ & \frac{1}{2} \sum_{j=0}^{2 n} \frac{\left(\begin{array}{c}
2 n \\
j
\end{array}\right)(2 n)_{j}(\gamma+1)_{j}}{\left(\frac{1}{2}\right)_{j}\left(j+\gamma+\frac{3}{2}\right)_{j}} \times \\
& { }_{3} F_{2}\left(\begin{array}{c}
j-2 n, j+2 n, j+\gamma+1 \\
j+\frac{1}{2}, 2 j+\gamma+\frac{5}{2}
\end{array}\right.
\end{aligned}
$$

For the sake of reducing the ${ }_{3} F_{2}(1)$ in $(4.2)$, we employ computer algebra. First, set

$$
M_{i, n, \gamma}={ }_{3} F_{2}\left(\begin{array}{c|c}
-i, 4 n-i, \gamma-i+2 n+1 & 1 \\
-i+2 n+\frac{1}{2}, \gamma-2 i+4 n+\frac{5}{2} & 1
\end{array}\right) .
$$

If we apply the algorithm of Zeilberger, we obtain the following recurrence relation of order one which is satisfied by $M_{i, n, \gamma}$ :

$$
\begin{aligned}
& (2 \gamma-2 i+5)(2 i-4 n-3)\left(-4 i^{2}+2 i(2 \gamma+8 n+3)-(2 \gamma+3)(4 n+1)\right) \times \\
& (2 \gamma-2 i+8 n+5) M_{i-1, n, \gamma}+(2 i-4 n-1)(2 \gamma-4 i+8 n+5) \times \\
& (2 \gamma-4 i+8 n+7)\left(-6 \gamma-4 i^{2}+2 i(2 \gamma+8 n+7)-4 n(2 \gamma+7)-13\right) \times \\
& M_{i, n, \gamma}=0,
\end{aligned}
$$

with the initial value: $M_{0, n, \gamma}=1$.

The exact solution of (4.3) is given by

$$
\begin{aligned}
& { }_{3} F_{2}\left(\begin{array}{c}
-i, 4 n-i, \gamma-i+2 n+1 \\
-i+2 n+\frac{1}{2}, \gamma-2 i+4 n+\frac{5}{2}
\end{array} \mid 1\right) \\
= & \frac{(-1)^{i+1}\left(-i+\gamma+\frac{5}{2}\right)_{i-1}\left(4 i^{2}-2 i(2 \gamma+8 n+3)+(2 \gamma+3)(4 n+1)\right)}{2(2 i-4 n-1)\left(-2 i+4 n+\gamma+\frac{5}{2}\right)_{i}}
\end{aligned}
$$


and therefore the ${ }_{3} F_{2}(1)$ in $(4.2)$ can be written in the following reduced form

$$
\begin{aligned}
& { }_{3} F_{2}\left(\begin{array}{c|c}
j-2 n, j+2 n, \gamma+j+1 \\
j+\frac{1}{2}, \gamma+2 j+\frac{5}{2}
\end{array}\right. \\
& =\frac{(-1)^{j} \Gamma\left(\gamma+\frac{3}{2}\right)\left(2 \gamma+2 j(2 \gamma+2 j+3)-16 n^{2}+3\right)}{2(2 j+1) \Gamma\left(j-2 n+\gamma+\frac{5}{2}\right)\left(2 j+\gamma+\frac{5}{2}\right)_{2 n-j}} .
\end{aligned}
$$

Now, the linearization formula (4.2) can be put in the following more simpler one:

$$
\begin{array}{r}
T_{n}^{2}(x)=\frac{1}{2}+\frac{1}{4} \sum_{j=0}^{2 n} \frac{(-1)^{j}\left(\begin{array}{c}
2 n \\
j
\end{array}\right) \Gamma\left(\gamma+\frac{3}{2}\right)(2 n)_{j}(\gamma+1)_{j}}{(2 j+1)\left(\frac{1}{2}\right)_{j}\left(j+\gamma+\frac{3}{2}\right)_{j}\left(2 j+\gamma+\frac{5}{2}\right)_{2 n-j}} \times \\
\frac{\left(2 \gamma+2 j(2 \gamma+2 j+3)-16 n^{2}+3\right)}{\Gamma\left(j-2 n+\gamma+\frac{5}{2}\right)} R_{j}^{\left(\gamma, \frac{1}{2}\right)}(x) .
\end{array}
$$

This completes the proof of Corollary 4.1 .

Now, the following two important formulae can be directly deduced as special cases of formula (4.1).

4.2. Corollary. If we set $\gamma=\frac{1}{2}$, and $\gamma=-\frac{1}{2}$, in the linearization formula (4.1), respectively, then the following two linearization formulae are obtained:

$$
\begin{aligned}
T_{n}^{2}(x) & =\frac{1}{4}\left(2-U_{2 n-2}(x)+U_{2 n}(x)\right), \\
T_{n}^{2}(x) & =\frac{1}{4}\left(2+V_{2 n-1}(x)+V_{2 n}(x)\right) .
\end{aligned}
$$

4.3. Remark. Noting the identity: $V_{j}(x)=(-1)^{j} W_{j}(x)$, it is easy to deduce the following linearization formula from (4.6).

$$
T_{n}^{2}(x)=\frac{1}{4}\left(2-W_{2 n-1}(x)+W_{2 n}(x)\right) .
$$

4.4. Remark. We note that the three formulae of the squares of $T_{n}(x)$ in (4.5), (4.6) and (4.7) can also follow from their trigonometric representations.

4.5. Corollary. For the case $\alpha=-\frac{1}{2}, \delta=\gamma+1$, the following linearization formula is obtained:

$$
\begin{aligned}
& T_{n}^{2}(x)=\frac{1}{2}+\frac{\sqrt{\pi} n \Gamma\left(\gamma+\frac{3}{2}\right)}{2^{2 \gamma+2} \Gamma(\gamma+1)} \times \\
& \left\{\sum_{m=0}^{n} \frac{(-1)^{m+n}(m+n-1) ! \Gamma(2 m+2 \gamma+2)}{(2 m) !(n-m) ! \Gamma\left(m-n+\gamma+\frac{3}{2}\right) \Gamma\left(m+n+\gamma+\frac{3}{2}\right)} R_{2 m}^{(\gamma, \gamma+1)}(x)\right. \\
& +\sum_{m=0}^{n-1} \frac{(-1)^{m+n+1}(m+n) ! \Gamma(2 m+2 \gamma+3)}{(2 m+1) !(n-m-1) ! \Gamma\left(m-n+\gamma+\frac{5}{2}\right) \Gamma\left(m+n+\gamma+\frac{5}{2}\right)} \times \\
& \left.R_{2 m+1}^{(\gamma, \gamma+1)}(x)\right\} .
\end{aligned}
$$

Proof. If we substitute by $\alpha=\frac{-1}{2}, \delta=\gamma+1$, then the linearization formula (3.1) is turned into

$$
\begin{aligned}
T_{n}^{2}(x)=\frac{1}{2}+ & \frac{1}{2} \sum_{j=0}^{2 n} \frac{\left(\begin{array}{c}
2 n \\
j
\end{array}\right)(2 n)_{j}(\gamma+1)_{j}}{\left(\frac{1}{2}\right)_{j}(j+2 \gamma+2)_{j}} \times \\
& { }_{3} F_{2}\left(\begin{array}{c}
j-2 n, j+2 n, j+\gamma+1 \\
j+\frac{1}{2}, 2 j+2 \gamma+3
\end{array}\right.
\end{aligned}
$$


Now, if we set

$$
M_{i, n, \gamma}={ }_{3} F_{2}\left(\begin{array}{c|c}
-i, 4 n-i, \gamma-i+2 n+1 & 1 \\
-i+2 n+\frac{1}{2}, 2 \gamma-2 i+4 n+3 & 1
\end{array}\right),
$$

then with the aid of Zeilberger's algorithm (see for instance, Koepf [21]), via the Maple software, and in particular, sumrecursion command, it can be shown that the following recurrence relation of order two is satisfied by $M_{i, n, \gamma}$ :

$$
\begin{aligned}
& (1-i)(2 \gamma-i+4)(i-4 n-1)(2 \gamma-2 i+4 n+3)(2 \gamma-i+4 n+4) \times \\
& M_{i-2, n, \gamma}+\left(-2 \gamma-2 i^{2}+4 \gamma i+8 n(-\gamma+i-2)+8 i-16 n^{2}-3\right) \times \\
& (-2 i+4 n+3)(2 \gamma-2 i+4 n+5) M_{i-1, n}+(2 i-4 n-3) \times \\
& (2 i-4 n-1)(2 \gamma-2 i+4 n+3)(2 \gamma-2 i+4 n+5)^{2} M_{i, n, \gamma}=0,
\end{aligned}
$$

with the initial values

$$
M_{0, n, \gamma}=1, \quad M_{1, n, \gamma}=\frac{1}{4 n+2 \gamma+1} .
$$

The exact solution of the recurrence relation (4.10) is:

$$
M_{i, n, \gamma}= \begin{cases}\frac{(-1)^{\frac{i}{2}} i !\left(\frac{3-i}{2}+\gamma\right)_{\frac{i}{2}}}{2^{i}\left(\frac{i}{2}\right) !\left(-i+2 n+\frac{1}{2}\right)_{\frac{i}{2}}\left(-i+2 n+\gamma+\frac{3}{2}\right)_{\frac{i}{2}}}, & i \text { even, } \\ \frac{(-1)^{\frac{i-1}{2}}(i+1) !\left(\frac{4-i}{2}+\gamma\right)_{\frac{i-1}{2}}}{2^{i+1}\left(\frac{i+1}{2}\right) !\left(-i+2 n+\frac{1}{2}\right)_{\frac{i-1}{2}}\left(-i+2 n+\gamma+\frac{3}{2}\right)_{\frac{i+1}{2}}}, & i \text { odd, }\end{cases}
$$

and therefore the ${ }_{3} F_{2}(1)$ in (4.9) has the following reduction formula

$$
\begin{aligned}
& { }_{3} F_{2}\left(\begin{array}{l|l}
j-2 n, j+2 n, j+\gamma+1 & 1 \\
j+\frac{1}{2}, 2 j+2 \gamma+3 & 1
\end{array}\right)=
\end{aligned}
$$

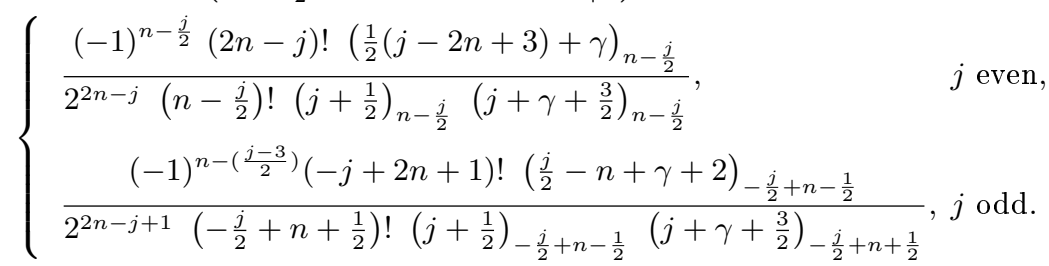

The last reduction formula enables one to write the linearization formula (4.9) in the form

$$
\begin{aligned}
& T_{n}^{2}(x)=\frac{1}{2}+\frac{\sqrt{\pi} n \Gamma\left(\gamma+\frac{3}{2}\right)}{2^{2 \gamma+2} \Gamma(\gamma+1)} \times \\
& \left\{\sum_{m=0}^{n} \frac{(-1)^{m+n}(m+n-1) ! \Gamma(2 m+2 \gamma+2)}{(2 m) !(n-m) ! \Gamma\left(m-n+\gamma+\frac{3}{2}\right) \Gamma\left(m+n+\gamma+\frac{3}{2}\right)} R_{2 m}^{(\gamma, \gamma+1)}(x)\right. \\
& +\sum_{m=0}^{n-1} \frac{(-1)^{m+n+1}(m+n) ! \Gamma(2 m+2 \gamma+3)}{(2 m+1) !(n-m-1) ! \Gamma\left(m-n+\gamma+\frac{5}{2}\right) \Gamma\left(m+n+\gamma+\frac{5}{2}\right)} \times \\
& \left.R_{2 m+1}^{(\gamma, \gamma+1)}(x)\right\} .
\end{aligned}
$$

Corollary 4.5 is now proved.

4.6. Remark. It is worthy to note here that the exact solution (4.11) of the recurrence relation (4.10) can be obtained with the aid of any suitable symbolic algorithm. The 
algorithms of Petkovsek (see, Koepf [21]), or the improved version of van Hoeij [31] may be employed for this purpose.

\section{References}

[1] W.M. Abd-Elhameed. On solving linear and nonlinear sixth-order two point boundary value problems via an elegant harmonic numbers operational matrix of derivatives. CMES-Comp. Model. Eng., 101(3):159-185, 2014.

[2] W.M. Abd-Elhameed. New formulae for the linearization coefficients of some nonsymmetric Jacobi polynomials. Adv. Differ. Eq., 2015(1):1-13, 2015.

[3] W.M. Abd-Elhameed. New product and linearization formulae of Jacobi polynomials of certain parameters. Integr. Transf. Spec, 26(8):586-599, 2015.

[4] W.M. Abd-Elhameed, E.H. Doha, and H.M. Ahmed. Linearization formulae for certain jacobi polynomials. Ramanujan J., 39(1):155-168, 2016.

[5] W.M. Abd-Elhameed, E.H. Doha, and Y.H. Youssri. Efficient spectral-Petrov-Galerkin methods for third-and fifth-order differential equations using general parameters generalized jacobi polynomials. Quaest. Math., 36(1):15-38, 2013.

[6] G.E. Andrews, R. Askey, and R. Roy. Special Functions. Cambridge University Press, Cambridge, 1999.

[7] R. Askey and G. Gasper. Linearization of the product of Jacobi polynomials. III. Can. J. Math, 23:332-338, 1971.

[8] H. Bateman, A. Erdélyi, W. Magnus, F. Oberhettinger, and F.G. Tricomi. Higher Transcendental Functions, volume I. McGraw-Hill New York, 1953.

[9] H. Chaggara and W. Koepf. On linearization coefficients of Jacobi polynomials. Appl. Math. Lett., 23(5):609-614, 2010.

[10] E.H. Doha. On the connection coefficients and recurrence relations arising from expansions in series of Laguerre polynomials. J. Phys. A: Math. Gen., 36(20):5449-5462, 2003.

[11] E.H. Doha. On the construction of recurrence relations for the expansion and connection coefficients in series of Jacobi polynomials. J. Phys. A: Math. Gen., 37(3):657, 2004.

[12] E.H. Doha and W.M. Abd-Elhameed. Integrals of Chebyshev polynomials of third and fourth kinds: An application to solution of boundary value problems with polynomial coefficients. J. Contemp. Math. Anal., 49(6):296-308, 2014.

[13] E.H. Doha and W.M. Abd-Elhameed. New linearization formulae for the products of chebyshev polynomials of third and fourth kinds. Rocky Mt. J. Math., 46(2):443-460, 2016.

[14] E.H. Doha, W.M. Abd-Elhameed, and H.M. Ahmed. The coefficients of differentiated expansions of double and triple Jacobi polynomials. B. Iran. Math. Soc., 38(3):739-766, 2012.

[15] E.H. Doha and H.M. Ahmed. Recurrences and explicit formulae for the expansion and connection coefficients in series of Bessel polynomials. J. Phys. A: Math. Gen., 37(33):8045, 2004.

[16] K.T. Elgindy and K.A. Smith-Miles. Solving boundary value problems, integral, and integrodifferential equations using Gegenbauer integration matrices. J. Comput. Appl. Math., 237(1):307-325, 2013.

[17] J.L. Fields and J. Wimp. Expansions of hypergeometric functions in hypergeometric functions. Math. Comp., 15(76):390-395, 1961.

[18] G. Gasper. Linearization of the product of Jacobi polynomials I. Can. J. Math., 22:171-175, 1970.

[19] G. Gasper. Linearization of the product of Jacobi polynomials II. Can. J. Math., 22:582593, 1970.

[20] E.A. Hylleraas. Linearization of products of Jacobi polynomials. Math. Scand., 10:189-200, 1962.

[21] W. Koepf. Hypergeometric summation: An Algorithmic Approach to Summation and Special Function Identities. Braunschweig, Germany: Vieweg, 1998.

[22] Y.L. Luke. The special $\mathrm{F}$ unctions and Their Approximations. Academic press, New York, 1969.

[23] C. Markett. Linearization of the product of symmetric orthogonal polynomials. Constr. Approx., 10(3):317-338, 1994. 
[24] J.C. Mason and D.C. Handscomb. Chebyshev Polynomials. Chapman and Hall, New York, NY, CRC, Boca Raton, 2010.

[25] F.W.J. Olver, D.W. Lozier, R.F. Boisvert, and C.W. Clark. NIST Handbook of Mathematical Functions. Cambridge University Press, 2010.

[26] M. Rahman. A non-negative representation of the linearization coefficients of the product of Jacobi polynomials. Can. J. Math., 33(4):915-928, 1981.

[27] E.D. Rainville. Special Functions. The Maximalan Company, New York, 1960.

[28] J. Sánchez-Ruiz. Linearization and connection formulae involving squares of Gegenbauer polynomials. Appl. Math. Lett., 14(3):261-267, 2001.

[29] J. Sánchez-Ruiz and J.S. Dehesa. Some connection and linearization problems for polynomials in and beyond the askey scheme. J. Comput. Appl. Math., 133(1):579-591, 2001.

[30] D.D. Tcheutia. On Connection, Linearization and Duplication Coefficients of Classical Orthogonal Polynomials. PhD thesis, Universität Kassel 2014. Available at https://kobra.bibliothek.uni-kassel.de/handle/urn:nbn:de:hebis:34-2014071645714., 2014.

[31] M. van Hoeij. Finite singularities and hypergeometric solutions of linear recurrence equations. J. Pure Appl. Algebra, 139(1):109-131, 1999.

[32] G.N. Watson. A note on generalized hypergeometric series. Proc. London Math. Soc, 2:23, 1925. 